\title{
A Metabolism Perspective on Pediatric Rhabdomyolysis
}

\section{(D) Havva Yazıcı, (D) Sema Kalkan Uçar}

Ege University Faculty of Medicine, Department of Pediatrics, Division of Metabolism and Nutrition, İzmir, Turkey

Cite this article as: Yazıcı H, Kalkan Uçar S. A Metabolism Perspective on Pediatric Rhabdomyolysis. Trends in Pediatrics 2021;2(4):147-153

\begin{abstract}
Rhabdomyolysis is a clinical emergency that can result in life-threatening complications. The etiology for rhabdomyolysis is broad. Infecitons are the most common cause in pediatric patients. Underlying inherited metabolic diseases are also a cause of rhabdomyolysis and can often have a diagnostic challenge, considering their marked heterogeneity and comparative rarity. The purpose of this review is to summarize the essential characteristics and diagnostic clues of inborn errors of metabolism associated with rhabdomyolysis.
\end{abstract}

Keywords: Rhabdomyolysis, inborn errors of metabolism, diagnosis

\section{INTRODUCTION}

Rhabdomyolysis is a clinical condition resulting from skeletal muscle fibers damage due to many reasons. The damaged muscle cell breaks down, and the intracytoplasmic proteins such as creatine kinase (CK) and myoglobin are released into the plasma. Subsequently, it can cause life-threatening complications such as electrolyte disturbance, acute kidney injury (AKI), and disseminated intravascular coagulation. These causes include severe trauma, vigorous exercise, burns, electrical injury, prolonged immobilization, seizure, electrolyte imbalances such as hypokalemia, hyponatremia, hypophosphatemia, and drugs such as levetiracetam, colchicine, lithium, and infectious agents such as Mycoplasma pneumoniae, enteroviruses, Human parainfluenza viruses (Table 1). ${ }^{1,2}$

Although there is a lack of consensus for diagnosis, in the pediatric literature, CK level greater than five times the upper limit of normal, or greater than $1.000 \mathrm{U} / \mathrm{L}$, is commonly used for diagnosis. Myalgia, weakness, and red urine are common complaints with elevated CK. This differs from myositis, in which there is muscle inflammation. However, the cell wall remains intact, so minimal intracellular content leaks into the circulation, and the serum CK level is much lower than $1.000 \mathrm{U} /$ L. $^{1-3}$

If rhabdomyolysis is suspected, the physician can confirm the diagnosis by detecting elevated serum CK levels. Rhabdomyolysis is a cause of red urine (Table 2). In the absence of red blood cells in the sediment, Heme-positive urine should raise the suspicion for hemolysis or rhabdomyolysis. Detecting urine myoglobin can be helpful for the diagnosis of rhabdomyolysis., ${ }^{4,5}$ Early recognition of rhabdomyolysis by pediatricians is vital because it can be encountered oftenly in the pediatric routine due to various etiologies and is mortal when there is delay in treatment. ${ }^{6}$

Inborn errors of metabolism (IEM) are also one of the main topics in the etiology of rhabdomyolysis. These conditions are listed in Table 3. IEMs may be overlooked, considering the high frequency of viral infections in pediatrics and the fact that these infections are the most common causes of rhabdomyolysis. Pediatricians should consider IEMs when symptoms are recurrent, progressive, unexplained, unresponsive to standard treatment, or inexplicably associated with the involvement of other organ systems. Consanguinity and patients who have similar clinical features in

S. Kalkan Uçar: 0000-0001-9574-7841

\section{Address for Correspondence: Havva Yazıc}

Ege University Faculty of Medicine, Department of Pediatrics, Division of Metabolism and Nutrition, İzmir, Turkey

E-mail: havvaya@gmail.com ORCID-ID: orcid.org/0000-0002-2564-7420

Received: 12.11.2021 Accepted: 19.12.2021 Publication date: 04.01.2022

${ }^{\circledR}$ Copyright 2021 by the Aydın Pediatric Society / Trends in Pediatrics published by Galenos Publishing House.

Licenced by Creative Commons Attribution 4.0 International (CC BY) 
family history are other supportive findings. ${ }^{3,7}$ This review aims to provide pediatricians with an overview of IEMs that can present with rhabdomyolysis. A summary of these conditions with a focus on diagnostic tools is presented in Figure $1 .^{8}$

\section{Glycogen Storage Diseases}

Glycogen storage diseases (GSDs) are inherited carbohydrate metabolism errors and are classified into two groups as hepatic and muscle glycogenoses. Hepatomegaly, hypoglycemia, elevated transaminases, and hepatosteatosis are common findings in hepatic glycogenosis. Glycogen is an important energy source for

\begin{tabular}{|c|c|}
\hline \multirow{8}{*}{ Infections } & Mycoplasma pneumoniae \\
\hline & Primary (acute) q fever \\
\hline & Enterovirus \\
\hline & Human parainfluenza viruses \\
\hline & West Nile encephalitis \\
\hline & Inflammatory bowel disease \\
\hline & Bacillus cereus \\
\hline & COVID-19 \\
\hline \multirow{4}{*}{$\begin{array}{l}\text { Fluid and electrolyte } \\
\text { imbalances }\end{array}$} & Water intoxication \\
\hline & Hypokalemia \\
\hline & Hyponatremia \\
\hline & Hypophosphatemia \\
\hline \multirow{4}{*}{$\begin{array}{l}\text { Muscle strain/excessive } \\
\text { activity/trauma }\end{array}$} & Burns \\
\hline & Prolonged immobilization \\
\hline & Electrical injury \\
\hline & Blunt trauma and crush injuries \\
\hline \multirow{7}{*}{ Medications } & Propofol \\
\hline & Cholesterol-lowering drugs \\
\hline & Levetiracetam \\
\hline & Daptomycin \\
\hline & Colchicine \\
\hline & Lithium \\
\hline & Synthetic marijuana \\
\hline \multirow{7}{*}{$\begin{array}{l}\text { Inherited } \\
\text { neuromuscular } \\
\text { disorders }\end{array}$} & Malignant hyperthermia-susceptibility \\
\hline & Anoctaminopathy-5 \\
\hline & Duchenne muscular dystrophy \\
\hline & Becker muscular dystrophy \\
\hline & $\begin{array}{l}\text { Limb-girdle muscular dystrophy (2B and } \\
2 \mathrm{I})\end{array}$ \\
\hline & Marinesco-Sjogren syndrome \\
\hline & Pontocerebellar hypoplasia type 2 \\
\hline $\begin{array}{l}\text { Inborn errors of } \\
\text { metabolism }\end{array}$ & (see Table 3) \\
\hline
\end{tabular}

skeletal muscle during exercise. The absence of enzymes involved in glycogenolysis causes defective glycogen breakdown and results in exercise intolerance, muscle cramps, and rhabdomyolysis in muscle glycogenosis. If the absent enzyme is also en enzyme expressed in tissues other than liver and skeletal muscle, other clinical manifestations such as neutropenia, hemolytic anemia, or proximal tubular dysfunction may occur depending on the affected tissue. The first diagnostic tool is, if available, a demonstration of glycogen storage and enzyme deficiency in the liver or muscle. Molecular analyses confirm the diagnosis. ${ }^{9,10}$

\section{GSD type V (Myophosphorylase deficiency, McArdle disease)}

The GSD type $\mathrm{V}$ is the most common and well-known type of muscle glycogenosis. GSD type $V$ is also known as McArdle disease. Myophosphorylase deficiency results in defective glycogenolysis in skeletal muscle. Typical symptoms are exercise intolerance with myalgia and stiffness of exercising muscles, which are relieved by rest. This clinical issue is named "second wind phenomenon". Two types of effort are more responsible for symptoms: brief, intense isometric exercise, such as lifting heavy weights, or constant

\begin{tabular}{|c|c|}
\hline \multicolumn{2}{|l|}{ Heme positive } \\
\hline \multirow{3}{*}{ Hemoglobinuria } & Hemolytic anemias \\
\hline & Hemolytic-uremic syndrome \\
\hline & Cystitis \\
\hline Myoglobinuria & Rhabdomyolysis \\
\hline \multicolumn{2}{|l|}{ Heme negative } \\
\hline \multirow{14}{*}{ Drugs } & Adriamycin \\
\hline & Chloroquine \\
\hline & Deferoxamine \\
\hline & Hydroxycobalamin \\
\hline & Ibuprofen \\
\hline & Iron sorbitol \\
\hline & Levodopa \\
\hline & Metronidazole \\
\hline & Nitrofurantoin \\
\hline & Phenytoin \\
\hline & Quinine \\
\hline & Rifampin \\
\hline & Salicylates \\
\hline & Sulfasalazine \\
\hline \multirow{4}{*}{ Foods } & Beets \\
\hline & Blackberries \\
\hline & Paprika \\
\hline & Red food coloring \\
\hline Porphyrinuria & Disorders of "Heme" metabolism \\
\hline
\end{tabular}


dynamic exercise, such as running fastly or climbing uphill..$^{9,11}$ Clinical examination is usually normal between rhabdomyolysis episodes, but a proximal muscle or scapulohumeral weakness and wasting may occur in the fourth decade of life. Baseline CK levels are high, and CK can increase to more than 100,000$1,000.000 \mathrm{UI} / \mathrm{L}$ during episodes of rhabdomyolysis, causing a

\begin{tabular}{|c|c|}
\hline \multicolumn{2}{|c|}{$\begin{array}{l}\text { Table 3. Inborn errors of metabolism to consider in } \\
\text { rhabdomyolysis }\end{array}$} \\
\hline \multirow{2}{*}{$\begin{array}{l}\text { Glycogen storage } \\
\text { diseases }\end{array}$} & GSD type $\mathrm{V}$ \\
\hline & GSD type Ixd \\
\hline \multirow{4}{*}{ Disorders of glycolysis } & ALDOA deficiency \\
\hline & LDH deficiency \\
\hline & Muscle phosphofructokinase deficiency \\
\hline & PGK deficiency \\
\hline \multirow{3}{*}{$\begin{array}{l}\text { Disorders of } \\
\text { mitochondrial fatty acid } \\
\text { oxidation }\end{array}$} & Carnitine transporter deficiency \\
\hline & $\begin{array}{l}\text { Carnitine palmitoyltransferase II } \\
\text { deficiency }\end{array}$ \\
\hline & VLCAD deficiency \\
\hline \multicolumn{2}{|c|}{ Mitochondrial disorders OXPHOS deficiencies } \\
\hline \multirow{2}{*}{ Others } & Lipin-1 deficiency \\
\hline & TANGO2 \\
\hline \multicolumn{2}{|c|}{$\begin{array}{l}\text { ALDOA: Aldolase A, GSD: Glycogen storage disease, LDH: Lactate dehydrogenase, } \\
\text { PGK: Phosphoglycerate kinase, VLCAD: Very long-chain acyl-CoA dehydrogenase, } \\
\text { OXPHOS: Oxidative phosphorylation system, TANGO2: Transport and Golgi } \\
\text { organization } 2\end{array}$} \\
\hline
\end{tabular}

risk of developing AKI. Myoglobinuria occurs in about half of the patients. The ischaemic forearm exercise test (IFET) was first used as a diagnostic tool. The abnormal increase in ammonia and the absence of lactate elevation during exercise support GSD-V diagnosis. Due to the risk of rhabdomyolysis, the standardized non-ischaemic FET has replaced the ischaemic test. Muscle biopsy shows storage and negative myophosphorylase staining. Muscle biopsy should be performed several weeks after a rhabdomyolysis episode. Recently, muscle biopsy may be avoided if sequencing of PYGM gene is available. ${ }^{10}$

There is no grateful therapy for GSD-V. Oral sucrose, ribose, or glucose ingestion together with abundant hydration before exercise is recommended to prevent rhabdomyolysis. ${ }^{12,13}$ Recently, a pilot study showed that a modified ketogenic diet might improve symptoms and exercise tolerance in patients with GSD-V. ${ }^{12}$

\section{GSD type IXd (Muscle phosphorylase kinase deficiency)}

GSD type IXd is caused by muscle phosphorylase kinase deficiency. Muscle phosphorylase kinase deficiency is an X-linked disorder caused by mutations in PHKA1, which encodes the $\alpha 1$ subunit. GSD-IXd is a cause of cramps, exercise intolerance and raised CK. The second wind phenomenon occurs. These symptoms are similar to GSD-V. They are usually milder and present anytime from childhood to adulthood. Rhabdomyolysis has been reported in patients with GSD-IXd. There is a variable lactate response with the non-ischaemic FET. Symptoms should be managed as in other muscle glycogenosis. ${ }^{10,13,14}$

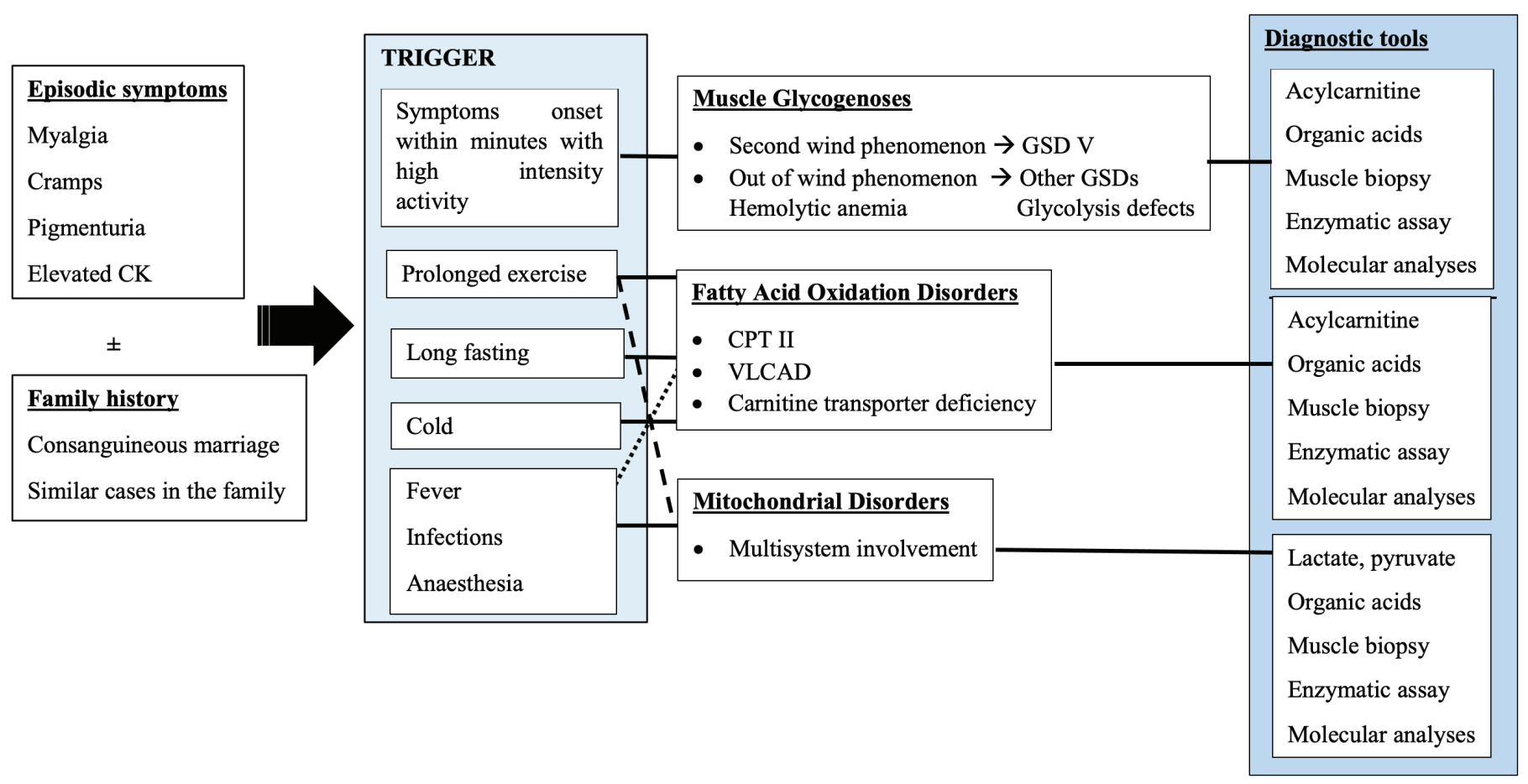

Figure 1. Algorithm for metabolic etiologies of rhabdomyolysis

CK: Creatine kinase, GSD: Glycogen storage disease, CPT: Carnitine palmitoyltransferase, VLCAD: Very long-chain acyl-CoA dehydrogenase 


\section{Disorders of Glycolysis}

\section{ALDOA deficiency (GSD type XII)}

Aldolase catalyzes the reversible conversion of fructose-1,6bisphosphate to glyceraldehyde 3-phosphate in glycolysis. Aldolase A (ALDOA) is a predominant isoform of aldolase in skeletal muscle and erythrocytes. Single cases with very variable symptoms are reported very rarely. The main clinical findings are hemolytic anemia, recurrent episodes of rhabdomyolysis, usually precipitated by fever, and mental retardation. CK levels are normal or elevated at rest. Muscle biopsy usually reveals nonspecific alterations and biochemical studies performed in muscle or erythrocytes show diminished ALDOA activity. ${ }^{15}$

There is no specific treatment. Avoiding vigorous exercise that may cause rhabdomyolysis is the main principle of management. ${ }^{16}$

\section{LDH deficiency (GSD type XI)}

Lactate dehydrogenase (LDH) has two subunits, namely muscle $(\mathrm{M})$ and heart $(\mathrm{H})$. There are five isoenzymes (LDH1-5) consisting of these two subunits. $\angle D H A$ and $\angle D H B$ genes encode the $\mathrm{M}$ and $\mathrm{H}$ proteins, respectively. LDHA mutations result in the deficiency of the LDH and they are very rare. It mainly affects skeletal muscles because skeletal LDH has all M-subunits. ${ }^{17}$ In addition to muscle symptoms, a few affected patients suffered from skin rashes. ${ }^{16,18}$ In patients with myoglobinuria, LDH deficiency should be kept in mind in the presence of low LDH despite elevated CK. ${ }^{16}$

\section{Muscle phosphofructokinase deficiency (GSD type VII, Tarui disease)}

There are three isoforms of phosphofructokinase; muscle, liver, and platelet isoforms. Muscle phosphofructokinase has the main role in glycolysis and the conversion of fructose 6-phosphate to fructose 1,6-bisphosphate. In GSD-VII, there is blockage of glycolysis resulting from a deficiency of the enzyme muscle phosphofructokinase due to mutations in the PFKM gene. The main clinical symptom is exercise intolerance, same as in GSD-V. The onset of classic form is usually in childhood. The main clinical features are muscle cramps, exercise intolerance, rhabdomyolysis, and myoglobinuria, often associated with hemolytic anemia and hyperuricemia but without the second wind phenomenon. Patients with late-onset form suffer from myalgia later in life. Infantile onset form may present as floppy infants. Infantile onset patients have no hemolytic anemia. ${ }^{11,16}$

The CK at rest is typically raised in GSD-V but may be normal in GSD-VII. A flat lactate curve, a normal increase of ammonia in nonischaemic FET, and increased bilirubin and reticulocyte counts are helpful for differentiation from GSD-V. Biochemical assay of the muscle phosphofructokinase level and PFKM gene analysis are required for diagnosis. ${ }^{11,16}$

No specific treatment exists. The patients should avoid a high carbohydrate diet and strenuous exercise, as glucose cannot be metabolized. According to a recent, 5-year follow-up study, ketogenic diet can benefit. ${ }^{19}$

\section{Muscle Phosphoglycerate Kinase Deficiency}

Phosphoglycerate kinase (PGK) deficiency is a rare X-linked metabolic disorder caused by mutations in the PGK1 gene. There are three main clinical presentations; non-spherocytic hemolytic anemia, myopathy, or the combination of anemia and central nervous system involvement. The myopathic form is indistinguishable from PFK deficiency and is characterized by recurrent episodes of exercise-induced cramps and myoglobinuria. The onset is usually in childhood.

Definitive diagnosis requires a biochemical assay of the PGK enzyme activity in muscle and/or erythrocytes and PGK1 gene analysis. ${ }^{20}$

No specific treatment or cure exists. Management primarily consists of avoiding strenuous exercise. Symptoms typically resolve with rest. ${ }^{16}$

\section{Disorders of Mitochondrial Fatty Acid Oxidation}

\section{Carnitine transporter deficiency}

Carnitine transporter deficiency, also called systemic carnitine deficiency, is caused by a lack of the sodium-dependent carnitine transporter protein OCTN2. Carnitine cannot pass through the plasma membrane. Therefore, entry of Acyl-CoA esters, transported by binding to carnitine, into the mitochondrial fatty acid oxidation cycle is disrupted. Carnitine which cannot be transported into the cell is lost through the kidneys, and plasma and intracellular carnitine levels decrease. For plasma, carnitine levels of patients are $<5 \mu \mathrm{M} / \mathrm{L}$, while the normal range is $25-50$ $\mu \mathrm{M} / \mathrm{L}$. The most fundamental clinical features are progressive cardiomyopathy and myopathy. Hypoglycemia and encephalopathy attacks may occur. It can cause sudden infant death. Older children have rhabdomyolysis, even in the absence of myopathy and cardiomyopathy. Asymptomatic or mild patients diagnosed by using neonatal screening were reported.Plasma carnitine level is tried to be kept above $10 \mu \mathrm{M} / \mathrm{L}$ with high-dose carnitine support $\left(100-300 \mathrm{mg} / \mathrm{kg} /\right.$ day). Response to treatment is good. ${ }^{21,22}$

\section{Carnitine palmitoyltransferase II deficiency}

Long-chain fatty acids do not cross the bilayer mitochondrial membrane. Thus, carnitine palmitoyltransferase (CPT) I and II afford long-chain fatty acids transport into the mitochondrial compartment. CPT II protein is in the inner mitochondrial membrane. CPT II deficiency is an autosomal recessive disorder of long-chain fatty acid oxidation.

There are three clinical phenotypes in CPT II deficiency; lethal neonatal form, severe infantile hepatocardiomuscular form, and myopathic form. ${ }^{23}$ Lethal neonatal form manifests with hypoketotic hypoglycemia, liver failure, cardiomyopathy, respiratory distress, and/or cardiac arrhythmias. Liver and brain calcifications, cystic dysplastic kidneys, and neuronal migration defects have been reported. ${ }^{24,25}$ Severe infantile hepatocardiomuscular form presents with hypoketotic hypoglycemia, liver failure, cardiomyopathy, and peripheral myopathy. ${ }^{25,26}$ 
The myopathic form is usually mild and can manifest from infancy to adulthood. The unbalanced sex distribution was reported in previous studies. More than $75 \%$ of patients reported were male thus far. ${ }^{27}$ It is clinically characterized by recurrent episodes of muscle pain, muscle weakness, and rhabdomyolysis. These episodes are triggered mainly through exercise, prolonged fasting, exposure to cold, fever, infection, menstruation, emotional stress. Affected individuals generally do not have muscle weakness in between the attacks. Renal failure requiring hemodialysis due to acute tubular necrosis is also occasionally reported during rhabdomyolysis attacks. The overall increase of C12 to C18 acylcarnitines will support the diagnosis of CPT II deficiency. The increases in C16+C18: 1 and $(\mathrm{C} 16+\mathrm{C} 18: 1) / \mathrm{C} 2$ ratios are more specific than other acylcarnitine measurements. The absence of the typical acylcarnitine profile does not exclude the diagnosis of CPT II deficiency, especially the mild myopathic form. ${ }^{28,29}$ Decreased CPT II activity and histopathological changes consisting lipid accumulation can be detected in muscle biopsy. Previous reports showed that muscle biopsies might be normal and showed non-specific changes or lipid deposition. ${ }^{30}$ The diagnosis should be confirmed by CPT // gene analysis. ${ }^{23} \mathrm{CPT}$ II deficient patients should avoid prolonged fasting and excessive muscle exercise in longterm treatment. Dietary therapy should be suggested to provide energy based on fractionated meals rich in carbohydrates and medium-chain triglyceride (MCT). ${ }^{31}$

\section{Very long-chain acyl-CoA dehydrogenase deficiency}

Very long-chain acyl-CoA dehydrogenase (VLCAD) plays a role in mitochondrial $\beta$-oxidation of long-chain fatty acids. There are three phenotypes reported. The severe or early-onset form typically presents within the first few months of life. It is characterized by hypertrophic or dilated cardiomyopathy and arrhythmias, hypotonia, hepatomegaly, and intermittent hypoglycemia. The moderate form typically presents during late infancy or early childhood with episodes of hypoketotic hypoglycemia and hepatomegaly. Cardiomyopathy is much less likely in moderate than in severe phenotypes. The mild or late-onset form is typically present in adolescence or early adulthood and is characterized by exercise intolerance and rhabdomyolysis. Episodic rhabdomyolysis may be provoked by infection, cold, fasting, exercise, or emotional stress. ${ }^{22,25}$ The specific marker for VLCAD from dried blood spots is an elevation of C14:1 acylcarnitine. Other long-chain acylcarnitines and/or abnormal ratios of C14:1 and other acylcarnitines support the diagnosis of VLCAD deficiency. The acylcarnitine profile can be completely normal in patients with mild VLCAD, which should be kept in mind. There is usually dicarboxylic aciduria, especially during severe metabolic decompensation. Muscle biopsy may show lipid storage, and the electromyography is often myopathic. Clinical and acylcarnitine profiles may be confused with CPT II deficiency. Enzyme analysis can be done for diagnosis. Diagnosis needs to be confirmed by enzyme assay of VLCAD or by mutation analysis of ACADVL encoding VLCAD. ${ }^{25}$

Prolonged fasting should be avoided in patients in order to prevent acute metabolic decompensation. Frequent, regular feeds which are high carbohydrate-low fat are recommended. For providing a source of energy; $\mathrm{MCT}$, by-passing the enzymatic block in $\beta$-oxidation is preferable. A bolus of MCT before exercise can prevent rhabdomyolysis in patients with myopathic VLCAD deficiency. ${ }^{25,32}$ Triheptanoin (C7 odd-chain fatty acid) can substitute for MCT in patients with VLCAD deficiency. ${ }^{33}$

Rhabdomyolysis attacks may result in life-threatening events such as acute renal failure requiring hemodialysis. Generally, the treatment of rhabdomyolysis is conservative such as intravascular volume expansion, urinary alkalinization. ${ }^{34}$

\section{Mitochondrial oxidative phosphorylation system disorders}

The oxidative phosphorylation system (OXPHOS) is the final step in the aerobic production of adenosine triphosphate. The OXPHOS consists of 5 protein complexes (Complex I-V) and 2 electron carriers embedded in the inner mitochondrial membrane. Defects in OXPHOS can be caused by a mutation in either mitochondrial DNA or nuclear DNA.

Most patients with mitochondrial disorders present with a multisystem disorder at any age, and the symptoms are almost progressive. The most commonly affected organs are the brain, kidney, heart, and skeletal muscle, all of which require the most energy. ${ }^{35,36}$

Mitochondrial myopathies lead to muscle energy failure due to dysfunction of the mitochondrial respiratory chain, coded by both mitochondrial and nuclear genome. It can be a non-progressive disease, but life-threatening episodes of rhabdomyolysis may occur. There is no effective treatment available for mitochondrial myopathies. ${ }^{37-39}$

\section{Others}

\section{Muscle phosphatidic acid phosphatase deficiency (Lipin-1 deficiency)}

Lipin-1 (LPIN1) is a phosphatidic acid phosphohydrolase that catalyzes the dephosphorylation of phosphatidic acid to diacylglycerol and inorganic phosphate. It regulates critical metabolic pathways, such as adipocyte differentiation and lipid metabolism, nuclear envelope and mitochondrial dynamics, and vacuole fusion. LPIN1 encodes Lipin-1.

LPIN1 deficiency is one of the leading metabolic causes of recurrent rhabdomyolysis episodes in children. Deficiency of this enzyme causes potentially fatal rhabdomyolysis triggered by infection. It should be kept in mind if normal acylcarnitines are detected in patients with recurrent rhabdomyolysis. Despite the known roles of LPIN1 in lipid biosynthesis and transcriptional regulation, the pathogenic mechanisms causing rhabdomyolysis are unclear.

There is no known effective treatment for LPIN1 deficiency. Symptomatic treatment of rhabdomyolysis, high energy intake from carbohydrates, and monitoring for hyperkalemia and cardiac arrhythmias are reported in the management of rhabdomyolysis. ${ }^{40-42}$ 


\section{TANGO2 Deficiency}

The Transport and Golgi Organization protein 2 (TANGO2) protein regulates the organization of the Golgi apparatus and the endoplasmic reticulum. Mutations in TANGO2 have been recently described in episodic rhabdomyolysis. Clinical findings are hypoglycemia, hyperammonemia, and susceptibility to life-threatening cardiac tachyarrhythmias. Neurologic abnormalities, developmental delay, and intellectual disability are other accompanying clinical courses of patients. Other clues for suspicion of TANGO2 deficiency are hypothyroidism, QT prolongation, or abnormalities of long-chain acylcarnitines and urine dicarboxylic acids. TANGO2 deficiency mimics fatty acid oxidation defects, except for ketosis..$^{43-45}$

There is no specific known treatment for TANGO2 deficiency. Treatment of rhabdomyolysis and organic complications (e.g., hyperammonemia, seizures, arrhythmias) is fundamental in managing attacks. Anabolizing management, treatment with vitamin B1, B2, and carnitine were reported to stabilize metabolic decompensation. ${ }^{46}$

\section{CONCLUSION}

Pediatricians should consider IEMs in the differential diagnosis of rhabdomyolysis. It should not be forgotten that IEMs have high prevalence due to the high rate of consanguineous marriages in our country. The road to diagnosis starts with the suspicion of the clinician and a thorough clinical evaluation.

\section{Ethics}

Peer-reviewed: Externally peer-reviewed.

Conflict of Interest: The authors declared no potential conflicts of interest with respect to the research, authorship, and/or publication of this article.

Funding: The authors received no financial support for the research, authorship, and/or publication of this article.

\section{REFERENCES}

1. Gardner HM, Askenazi DJ, Hoefert JA, Helton A, Wu CL. Acute kidney injury among children admitted with viral rhabdomyolysis. Hosp Pediatr. 2021;11:878-85.

2. Scalco RS, Gardiner AR, Pitceathly RD, et al. Rhabdomyolysis: a genetic perspective. Orphanet J Rare Dis. 2015;10:1-15.

3. Saudubray JM, Baumgartner M, Walter J. Clinical approach to inborn errors of metabolism in pediatrics. Inborn metabolic diseases. In: Saudubray J-M, Garcia-Cazorla A (eds). Inborn Metabolic Diseases. Springer, Berlin, Heidelberg, 2016;3-70.

4. Lockwood GM, Storm DW. Urine Testing in Children: Little People, Big Challenges. Urine Tests: Springer; 2020:259-80.

5. Pan C, Avner E. Clinical evaluation of the child with hematuria. Nelson textbook of pediatrics $20^{\text {th }}$ Int ed Elsevier Saunders, Philadelphia, $2016 ; 2494$.

6. Schiff M, Mochel F, Dionisi-Vici C. Emergency Treatments. In: Saudubray J-M, Garcia-Cazorla A (eds). Inborn Metabolic Diseases. Springer, Berlin, Heidelberg, 2016;109-17.

7. El-Gharbawy A, Vockley J. Inborn errors of metabolism with myopathy: defects of fatty acid oxidation and the carnitine shuttle system. Pediatr Clin North Am. 2018;65:317-35.
8. Chan EK, Kornberg AJ, Ryan MM. A diagnostic approach to recurrent myalgia and rhabdomyolysis in children. Arch Dis Child. 2015;100:793-7.

9. Scalco RS, Lucia A, Santalla A, et al. Data from the European registry for patients with McArdle disease and other muscle glycogenoses (EUROMAC). Orphanet J Rare Dis. 2020;15:330.

10. Walter J, Labrune PA, Laforet P. The glycogen storage diseases and related disorders. In: Saudubray J-M, Garcia-Cazorla A (eds). Inborn metabolic diseases. Springer, Berlin, Heidelberg, 2016;121-37.

11. Lucia A, Martinuzzi A, Nogales-Gadea G, Quinlivan R, Reason S. Clinical practice guidelines for Glycogen Storage Disease V \& VII (McArdle Disease and Tarui Disease) from an international study group. Neuromuscul Disord. 2021;31:1296-310.

12. Løkken N, Hansen KK, Storgaard JH, Ørngreen MC, Quinlivan R, Vissing J. Titrating a modified ketogenic diet for patients with McArdle disease: $A$ pilot study. J Inherit Metab Dis. 2020;43:778-86.

13. Santer R, Klepper J, Smit GPA. Disorders of carbohydrate metabolism and glucose transport. In: Blau N, Duran M, Gibson KM, Dionisi-Vici K. Physician's Guide to the Diagnosis, Treatment, And Follow-Up Of Inherited Metabolic Diseases. Springer, Berlin, Heidelberg, 2014;265-301.

14. Kishnani PS, Goldstein J, Austin SL, et al. Diagnosis and management of glycogen storage diseases type VI and IX: a clinical practice resource of the American College of Medical Genetics and Genomics (ACMG). Genet Med. 2019;21:772-89.

15. Papadopoulos C, Svingou M, Kekou K, et al. Aldolase A deficiency: Report of new cases and literature review. Mol Genet Metab Rep. 2021;27:100730.

16. Wamelink MM, Valayannopoulos V, Garavaglia B. Disorders of glycolysis and the pentose phosphate pathway. In: Saudubray J-M, Garcia-Cazorla A (eds). Inborn Metabolic Diseases. Springer, Berlin, Heidelberg, 2016;14960.

17. Farhana A, Lappin S. Biochemistry, Lactate Dehydrogenase.[Updated 2020 May 17]. StatPearls [Internet] Treasure Island (FL): StatPearls Publishing 2021.

18. Kanno T, Maekawa M. Lactate dehydrogenase M-subunit deficiencies: clinical features, metabolic background, and genetic heterogeneities. Muscle Nerve Suppl. 1995;18:S54-S60.

19. Similä ME, Auranen M, Piirilä PL. Beneficial effects of ketogenic diet on phosphofructokinase deficiency (glycogen storage disease type VII). Front Neurol. 2020;11:57.

20. Echaniz-Laguna A, Nadjar Y, Béhin A, et al. Phosphoglycerate kinase deficiency: a nationwide multicenter retrospective study. J Inherit Metab Dis, 2019;42:803-8.

21. Longo N. Primary carnitine deficiency and newborn screening for disorders of the carnitine cycle. Ann Nutr Metab. 2016;68:5-9.

22. Spiekerkoetter $U$, Duran M. Mitochondrial fatty acid oxidation disorders. In: Blau N, Duran M, Gibson KM, Dionisi-Vici K. Physician's Guide to the Diagnosis, Treatment, And Follow-Up Of Inherited Metabolic Diseases. Springer, Berlin, Heidelberg, 2014;247-64.

23. Joshi PR, Zierz S. Muscle carnitine palmitoyltransferase II (CPT II) deficiency: a conceptual approach. Molecules. 2020;25:1784.

24. Boemer F, Deberg M, Schoos R, et al. Diagnostic pitfall in antenatal manifestations of CPT II deficiency. Clin Genet. 2016;89:193-7.

25. Morris AA, Spiekerkoetter U. Disorders of mitochondrial fatty acid oxidation \& riboflavin metabolism. In: Saudubray J-M, Garcia-Cazorla A (eds). Inborn metabolic diseases. Springer, Berlin, Heidelberg, 2016;20113.

26. Vladutiu GD, Quackenbush EJ, Hainline BE, Albers S, Smail DS, Bennett MJ. Lethal neonatal and severe late infantile forms of carnitine palmitoyltransferase II deficiency associated with compound heterozygosity for different protein truncation mutations. J Pediatr. 2002;141:734-6.

27. Joshi PR, Deschauer M, Zierz S. Carnitine palmitoyltransferase II (CPT II) deficiency: genotype-phenotype analysis of 50 patients. J Neurol Sci. 2014;338:107-11.

28. Gempel K, Kiechl S, Hofmann S, et al. Screening for carnitine palmitoyltransferase II deficiency by tandem mass spectrometry. J Inherit Metab Dis. 2002;25:17-27. 
29. Anichini A, Fanin M, Vianey-Saban C, et al. Genotype-phenotype correlations in a large series of patients with muscle type CPT II deficiency. Neurol Res. 2011;33:24-32.

30. Angelini C, Nascimbeni AC, Cenacchi G, Tasca E. Lipolysis and lipophagy in lipid storage myopathies. Biochimic Biophys Acta. 2016;1862:1367-73.

31. Ivin N, Della Torre V, Sanders F, Youngman M. Rhabdomyolysis caused by carnitine palmitoyltransferase 2 deficiency: a case report and systematic review of the literature. J Intensive Care Soc. 2020;21:165-73.

32. Van Calcar SC, Sowa M, Rohr F, et al. Nutrition management guideline for very-long chain acyl-CoA dehydrogenase deficiency (VLCAD): an evidenceand consensus-based approach. Mol Genet Metab. 2020;131:23-37.

33. Norris MK, Scott Al, Sullivan S, et al. Tutorial: triheptanoin and nutrition management for treatment of long-chain fatty acid oxidation disorders. JPEN J Parenter Enteral Nutr.2021;45:320-8.

34. Merritt JL, MacLeod E, Jurecka A, Hainline B. Clinical manifestations and management of fatty acid oxidation disorders. Rev Endocr Metab Disord 2020;21:479-93.

35. de Laat P, Rodenburg R, Smeitink J. Mitochondrial oxidative phosphorylation disorders. In: Blau N, Duran M, Gibson KM, Dionisi-Vici K. Physician's Guide to the Diagnosis, Treatment, And Follow-Up Of Inherited Metabolic Diseases. Springer, Berlin, Heidelberg, 2014;337-59.

36. Rahman S, Mayr JA. Disorders of oxidative phosphorylation. In: Saudubray J-M, Garcia-Cazorla A (eds). Inborn metabolic diseases. Springer, Berlin, Heidelberg, 2016;223-42.

37. Andreu A, Bruno C, Dunne T, et al. A nonsense mutation (G15059A) in the cytochrome $b$ gene in a patient with exercise intolerance and myoglobinuria. Annal Neurol. 1999;45:127-30.
38. Andreu $A L$, Hanna MG, Reichmann $H$, et al. Exercise intolerance due to mutations in the cytochrome $b$ gene of mitochondrial DNA. N Engl J Med. 1999;341:1037-44.

39. Olimpio C, Tiet MY, Horvath R. Primary mitochondrial myopathies in childhood. Neuromuscul Disord. 2021;31:978-87.

40. Pizzamiglio C, Lahiri N, Nirmalananthan N, et al. First presentation of LPIN1 acute rhabdomyolysis in adolescence and adulthood. Neuromuscul Disord. 2020;30:566-71.

41. Topal S, Demir M, Hasan A, et al. A neglected cause of recurrent rhabdomyolysis, LPIN1 gene defect: a rare case from Turkey. Turk J Pediatr Dis. 2020;62:647-51.

42. Lamari F, Saudubray J-M, Mitchell GA. Disorders of Intracellular Triglyceride and Phospholipid Metabolism In: Saudubray J-M, Garcia-Cazorla A (eds). Inborn metabolic diseases. Springer, Berlin, Heidelberg, 2016;477-91.

43. Schymick J, Leahy $P$, Cowan $T$, et al. Variable clinical severity in TANGO2 deficiency: Case series and literature review. Am J Med Genet A. 2021 Oct 19. doi: 10.1002/ajmg.a.62543. Epub ahead of print

44. Bérat CM, Montealegre S, Wiedemann A, et al. Clinical and biological characterization of 20 patients with TANGO2 deficiency indicates novel triggers of metabolic crises and no primary energetic defect. J Inherit Metab Dis. 2021;44:415-25.

45. Dines JN, Golden-Grant K, LaCroix A, et al. TANGO2: expanding the clinical phenotype and spectrum of pathogenic variants. Genet Med. 2019;21:601-7.

46. Mingirulli N, Pyle A, Hathazi D, et al. Clinical presentation and proteomic signature of patients with TANGO2 mutations. J Inherit Metab Dis. 2020;43:297-308. 